\title{
OPEN Retinal ganglion cell dysfunction in preclinical Alzheimer's disease: an electrophysiologic biomarker signature
}

\author{
Samuel Asanad ${ }^{1,2 \bowtie}$, Christian M. Felix ${ }^{3,4}{ }^{1,}$ Michele Fantini ${ }^{5}$, Michael G. Harrington ${ }^{6}$, \\ Alfredo A. Sadun ${ }^{1,4}$ \& Rustum Karanjia ${ }^{1,4,7,8}$
}

The current study evaluated retinal function using electroretinography (ERG) in cognitively healthy (CH) participants with preclinical Alzheimer's disease (AD), as classified by cerebral spinal fluid (CSF) $A \beta_{42} /$ Tau ratio. Individuals with normal retinal morphology ascertained by spectral-domain optical coherence tomography were enrolled. Full-field ERG, pattern PERG, and photopic negative response (PhNR) were performed in 29 adult participants (58 eyes). Amplitude and implicit times of the ERG wave components were analyzed. Preclinical AD participants showed marked retinal ganglion cell dysfunction relative to controls. The PhNR was significantly diminished in preclinical AD relative to controls. PhNR amplitude and N95 implicit time differentiated $\mathrm{CH}$ individuals with CSF biomarkers of AD pathology with $87 \%$ sensitivity and $82 \%$ specificity. These quantitative electrophysiologic findings expand our understanding of early retinal functional changes that precede cognitive decline in AD. Retinal ganglion cell dysfunction, as detected by ERG, may be a clinically useful, non-invasive in vivo biomarker for early disease detection, which is necessary for ultimately pursuing early intervention.

Alzheimer's disease (AD), a chronic neurodegenerative disorder, is the most common form of dementia, affects over 26 million people worldwide, and is the most expensive disease in the United States at a total projected cost of $\$ 305$ billion in $2020^{1,2}$. In addition to progressive memory loss and cognitive decline ${ }^{3}$, ophthalmologic impairments in contrast sensitivity, color discrimination, and motion perception have also been reported in $\mathrm{AD}^{4}$. Intriguingly, however, these symptoms of visual dysfunction in $\mathrm{AD}$ cannot be explained by cortical deficits alone and have been associated with the degeneration of anterior visual pathways, namely the optic nerve and the retina ${ }^{5-10}$. These ophthalmologic manifestations have not only been documented in the early stages of dementia, but even prior to presenting as a clear diagnosis of $\mathrm{AD}^{8-11}$.

Our laboratory provided the first histopathological evidence of optic neuropathy in AD after observing diffuse retinal ganglion cell (RGC) and axonal atrophy in postmortem optic nerve tissues derived from severe AD patients ${ }^{12}$. Pathologic hallmarks typical for $\mathrm{AD}$ in the brain have also been shown in the retina. We previously demonstrated $\mathrm{A} \beta$ accumulation inside and around melanopsin-immunoreactive RGCs (mRGCs) in postmortem $\mathrm{AD}$ eyes with corresponding degeneration of the retinal nerve fiber (RNFL) and ganglion cell layers (RGCL) ${ }^{8,13-18}$. These postmortem findings have been verified in vivo following the advent of optical coherence tomography (OCT). Live human studies have revealed significant retinal thinning in AD patients as well as in early-stage disease ${ }^{6,19-22}$. Nevertheless, the clinical utility of the retina as a biomarker for early AD remains limited ${ }^{23-28}$.

Pre-symptomatic $\mathrm{AD}$ describes cognitively healthy $(\mathrm{CH})$ individuals having $\mathrm{AD}$ pathology many years prior to symptom onset. The combined ratio of $A \beta_{42}$ and Tau in the cerebrospinal fluid (CSF) has been shown to predict $\mathrm{AD}$ neuropathology with higher accuracy relative to the performance of these proteins individually, suggesting that both amyloid and Tau likely contribute, independently, to disease pathogenesis ${ }^{29}$. In parallel with these findings, more recent studies have also shown that the earliest preclinical AD can also be detected with greater sensitivity and specificity by the $\mathrm{A} \beta_{42}$ /Tau ratio relative to the CSF concentrations of these biomarkers

${ }^{1}$ Doheny Eye Centers-UCLA, Pasadena, CA, USA. ${ }^{2}$ Department of Ophthalmology and Visual Sciences, University of Maryland School of Medicine, Baltimore, MD, USA. ${ }^{3}$ Charles R. Drew University of Medicine and Science, LoS Angeles, CA, USA. ' David Geffen School of Medicine, University of California, Los Angeles, Los Angeles, CA, USA. ${ }^{5}$ Department of Ophthalmology, Sacro Cuore Don Calabria Hospital, Negrar, Italy. ${ }^{6}$ Huntington Medical Research Institutes, Pasadena, CA, USA. 'Department of Ophthalmology, University of Ottawa, Ottawa, ON, Canada. ${ }^{8}$ Ottawa Hospital Research Institute, Ottawa, ON, Canada. ${ }^{\square}$ email: samuelasanad@gmail.com 
independently ${ }^{30}$. Notably, a recent prospective study conducted by our group demonstrated significant retinal thinning in preclinical AD participants who were biochemically confirmed for abnormal CSF A $\beta_{42} / \mathrm{Tau}^{31}$. In addition to structural abnormalities, various $\mathrm{AD}$ studies have also shown functional changes in the retina using electroretinography $(E R G)^{32-36}$. The present study evaluates, for the first time, retinal function using ERG in preclinical AD participants with structurally normal OCTs and investigates whether retinal electrophysiologic data can help discriminate between $\mathrm{CH}$ individuals with normal versus pathological $A \beta_{42} /$ Tau ratio.

\section{Methods}

Human participants. The Institutional Review Boards of both the Huntington Medical Research Institutes (HMRI), Pasadena and the University of California, Los Angeles (UCLA) approved the protocol and consent forms for this study, which was conducted and performed in compliance with the ethical standards set out in the Declaration of Helsinki. Prior to enrollment, all study participants gave written, informed consent. Clinical and CSF studies were performed at HMRI, and ophthalmology assessments were conducted at the Doheny Eye Centers, UCLA, Division of Neuro-ophthalmology, Pasadena, CA.

Participants over 60 years of age were recruited locally at HMRI for aging research if they had no cognitive impairment after medical and neuropsychological assessment, using the Uniform Data Set-3 criteria of the National Alzheimer's Coordinating Center (https://www.alz.washington.edu/WEB/npsych_means.html) and after consensus clinical conference. CSF $A \beta_{42} /$ Tau ratios were determined from lumbar fluid as described ${ }^{37}$. MSD assay (catalog no. K15199G, MSD, Rockville, MD) was used to determine CSF levels of A $\beta_{42}$. MSD assay (catalog no. K15121G, MSD, Rockville, MD) was used to determine CSF levels of total tau. We used a logistic regression cutoff for the $\mathrm{CSF} \mathrm{AB}_{42}$ /Tau ratio that correctly classified more than $85 \%$ of probable $\mathrm{AD}$ patients to identify two separate cohorts of the $\mathrm{CH}$ participants: those with Normal $\mathrm{A} \beta_{42} /$ Tau ratio ("CH-NAT"), or those with Pathological $\mathrm{AB}_{42}$ /Tau ratio ("CH-PAT"). We thus defined that $\mathrm{CH}-\mathrm{PAT}$ individuals as preclinical AD.

All subjects had a complete ophthalmic examination including assessment of best-corrected visual acuity (BCVA), intraocular pressure (IOP), slit lamp examination of the anterior segment, and a dilated fundus examination. Subjects were excluded from this study based on the following exclusion criteria: BCVA $<20 / 50$; refractive error $> \pm 5$ diopters, spherical equivalent; poor OCT image quality defined by an image signal less than 6 due to severe cataracts or unstable fixation; IOP $>20 \mathrm{~mm} \mathrm{Hg}$; pre-existing retinal pathologies such as retinal vascular occlusion or retinal dystrophy; pre-existing ocular diseases such as glaucoma, optic neuropathy or uveitis; previous intraocular surgery or laser treatment except for cataract surgery performed at least 12 months prior to enrollment; penetrating ocular trauma; current active smoking status; and history or evidence of other neurological or psychiatric disorders, diabetes mellitus, poorly controlled systemic arterial hypertension defined by systolic $>150$ and diastolic $>90$, cardiovascular diseases, renal failure, substance abuse in the past 5 years, systemic corticosteroid use exceeding 6 months, and systemic autoimmune conditions with associated optic neuropathies.

Retinal imaging. Retinal nerve fiber layer (RNFL), retinal ganglion cell-inner plexiform layer (RGC-IPL), and full macular thicknesses were measured for all participants using spectral-domain OCT (Cirrus SD-OCT, software v 6.0; Carl Zeiss Meditec). Scans were all acquired using the protocols for Optic Disc Cube $200 \times 200$ and the Macular Cube $512 \times 128$ in both eyes with pupil dilation. Following proper seating and alignment of each individual, the iris was brought into view using the mouse-driven alignment system, and the ophthalmoscopic image was focused. To acquire the Optic Disc Cube, the optic nerve head was centered on the live image, and centering and enhancement were optimized. After launching the scanning process, the instrument's $840 \mathrm{~nm}$ wavelength laser beam generated a cube of data measuring $6 \mathrm{~mm} \times 6 \mathrm{~mm}$ after scanning a series of 200 B-scans with $200 \mathrm{~A}$-scans per B-scan (40,000 points) in $1.5 \mathrm{~s}$ (27,000 A-scans/sec). Cirrus SD-OCT algorithms were used to find the optic disc with automatic placement of a calculation circle measuring $3.46 \mathrm{~mm}$ in diameter symmetrically around it. Layer seeking algorithms were used to find the RNFL inner (anterior) boundary and the RNFL outer (posterior) boundary for the entire cube except the optic disc. The system extracted $256 \mathrm{~A}$-scan samples from the data cube along the path of the calculation circle.

With respect to acquiring the Macular Cube, participants fixated on the central target. The ganglion cell analysis algorithm detected and measured the thickness of the macular RGC-IPL within a $14.13-\mathrm{mm}^{2}$ elliptical annulus area centered on the fovea. The ganglion cell analysis algorithm processed data from 3-dimensional volume scans and measured the thickness of the macular RGC-IPL. Retinal thickness maps of the macular region were acquired using the macular cube scan within a $6 \times 6-\mathrm{mm}^{2}$ circular area centered on the fovea. Measurements were averaged over 9 -retinal subfields, as defined by the Early Treatment Diabetic Retinopathy Study. A published, detailed description of this algorithm and how it operates is available for reference ${ }^{37}$. The built-in SD-OCT eye-tracking system provided reproducible measurements with a coefficient of variation of $0.5 \%{ }^{38}$. An experienced operator captured all images. Individual scan volumes were reviewed for segmentation errors. Scans with significant motion artifacts, segmentation errors, or signal strength values less than 6 were excluded from analysis. To maximize the reflective signal, polarization was optimized and the scan with the best centration of the optic disc was consistently selected.

Pattern electroretinogram. Transient PERG was performed to allow clear separation and evaluation of the P50 and N95 components. Examination was conducted according to the International Society for Clinical Electrophysiology of Vision (ISCEV) standards. ERGs were recorded with DTL-Plus (Diagnosys LLC, Lowell, MA, USA) microconductive thread electrodes with the fiber positioned at the lower cornea surface and secured on the temporal and nasal canthus after the application of topical anesthesia with proparacaine $0.5 \%$ (Anestalcon, Alcon, Fort Worth, TX, USA). Gold cup electrodes were placed on the temple for reference and central forehead $(\mathrm{Fz})$ for ground. 


\begin{tabular}{|l|l|l|l|}
\hline & CH-NAT & CH-PAT & P \\
\hline No. of Participants (eyes) & $14(28)$ & $15(30)$ & - \\
\hline Mean Age, years & $75.9 \pm 8.5$ & $76.5 \pm 6.6$ & 0.6 \\
\hline Women $($ Men $)$ & $10(4)$ & $12(3)$ & 0.4 \\
\hline RNFL Thickness $(\mu \mathrm{m})$ & $88.1 \pm 8.3$ & $84.24 \pm 7.4$ & 0.5 \\
\hline RGC-IPL Thickness $(\mu \mathrm{m})$ & $74.5 \pm 6.0$ & $74.1 \pm 5.8$ & 0.8 \\
\hline Macular Thickness $(\mu \mathrm{m})$ & $280.6 \pm 15.1$ & $272.1 \pm 14.9$ & 0.3 \\
\hline CSF A $\beta_{42} /$ Tau & $4.5 \pm 1.3$ & $1.3 \pm 0.4$ & $<\mathbf{0 . 0 0 0 1}$ \\
\hline
\end{tabular}

Table 1. Cohort demographics, retinal and cerebrospinal fluid (CSF) data. RNFL, retinal nerve fiber layer; RGC-IPL, retinal ganglion cell-inner plexiform layer. CH-NAT, cognitively healthy with normal amyloid/tau ratio; $\mathrm{CH}-\mathrm{PAT}$, cognitively healthy with pathologic amyloid/tau ratio. Data are provided as mean \pm standard deviation.

Electrical signals were recorded using the Envoy Monocular Pattern Stimulus (Diagnosys LLC, United States) with occlusion of the contralateral eye. Recordings were obtained from both right and left eyes. The stimulusgenerated black and white alternating contrast reversing bars (mean luminance, $50 \mathrm{~cd} / \mathrm{m}^{2}$; spatial frequency, 0.033 cycle/deg; contrast, 99\%; and temporal frequency, $1 \mathrm{~Hz}$ ) were recorded without pupil dilation and were viewed through appropriate refractive correction to maximize retinal image quality over the full stimulus field. Participants were seated $5 \mathrm{~cm}$ from the screen and were instructed to fixate on a target at the center of the OLED display. The artifact rejection system was employed to filter large potential caused by extraocular movement and eye blinks. A minimum of 200 sweeps were recorded and included for analysis. The standard, transient response separates the PERG into wave components including a negative wave at about $35 \mathrm{~ms}$ (N35) followed by a positive wave at approximately $50 \mathrm{~ms}$ (P50) and a large, negative wave at around $95 \mathrm{~ms}$ (N95). PERG waveforms were visually inspected, and the P50-wave, N95-wave components were determined. The P50 amplitude was measured from the trough of N35 to the peak of P50. The N95 amplitude was measured from the peak of P50 to the trough of N95.

Full-field electroretinogram. The ffERG was recorded with the same electrode montage as the PERG. Per ISCEV standards, the pupils were maximally dilated to approximately $8 \mathrm{~mm}$ in diameter following topical administration of $1 \%$ tropicamide and $10 \%$ phenylephrine. Stimuli were generated using the ColorDome ganzfeld associated with the Espion E3 system (Diagnosys LLC) with electrical signals being recorded simultaneously from both eyes. Participants were dark-adapted for a period of $20 \mathrm{~min}$ before recording the scotopic ERG responses (ISCEV standard dark-adapted 0.01 ERG, 3 ERG, 10 ERG). Following scotopic testing, participants were light-adapted for a period of $10 \mathrm{~min}$ before performing photopic ERG responses (ISCEV standard lightadapted 3 ERG and 30-Hz flicker). Recordings were averaged from 9 sweeps for each participant.

Photopic negative response. The PhNR stimulus conditions were produced by ColorDome ganzfeld (Diagnosys LLC). Red $(640 \mathrm{~nm})$ stimulus flashes of $4 \mathrm{~ms}$ duration were presented at a $4-\mathrm{Hz}$ rate on a blue $(470 \mathrm{~nm})$ rod saturating background. Red flash stimulation was presented at $1 \mathrm{~cd} \cdot \mathrm{s} / \mathrm{m}^{2}$, while the blue background remained at $10 \mathrm{~cd} / \mathrm{m}^{2}$. An Espion E3 was used to record PhNR waveforms. Six sets of 25 sweeps of 150ms duration were recorded with bandpass filtering between 0.3 and $300 \mathrm{~Hz}$ at the six stimulus flash intensities. Each of the six repetitions was manually filtered to remove eye blink and other motion artifacts, and an average of the remained responses was generated for each eye. Recordings were obtained from both right and left eyes. $\mathrm{PhNR}$ waveforms were visually inspected, and the a-wave, b-wave, and PhNR components were determined.

Statistical methods. Group comparison analysis of the ERG response was made using mixed model repeated measures with unstructured covariance. Group (CH-NAT, CH-PAT), ERG protocol (PERG, ffERG, PhNR), ERG parameters (P50, N95, a-wave, b-wave, PhNR, amplitude and implicit time), and eye laterality (OD, OS) were fixed effects; and age and gender were covariates.

Repeated measure multivariable logistic regression (Generalized Linear Mixed Model) based on the forward Wald stepwise procedure was used to examine the unique contributions of ERG wave-components in group classification of $\mathrm{CH}$-PATs while avoiding multicollinearity. Only subjects with all data points available (all ERG protocols and all wave-components for both eyes) were included. The final model constituted independently significant predictors. Receiver operating characteristic (ROC) curve analysis was used to assess the potential diagnostic ability of the binary classification system according to the area under the curve (AUC) and to derive a cutoff for predicted event probability. Statistical significance was assumed at $p<0.05$. Analysis was performed using SPSS V.20 package software.

\section{Results}

Fifteen participants were classified as $\mathrm{CH}-\mathrm{PAT}$ (mean age \pm standard deviation $76.5 \pm 6.6$ years) and 14 as $\mathrm{CH}$ NAT (mean age $75.9 \pm 8.5$ years). There were no differences between the groups for age and gender. There were no significant group differences for RNFL, GC-IPL or macular full-thicknesses as measured by SD-OCT (Table 1). 


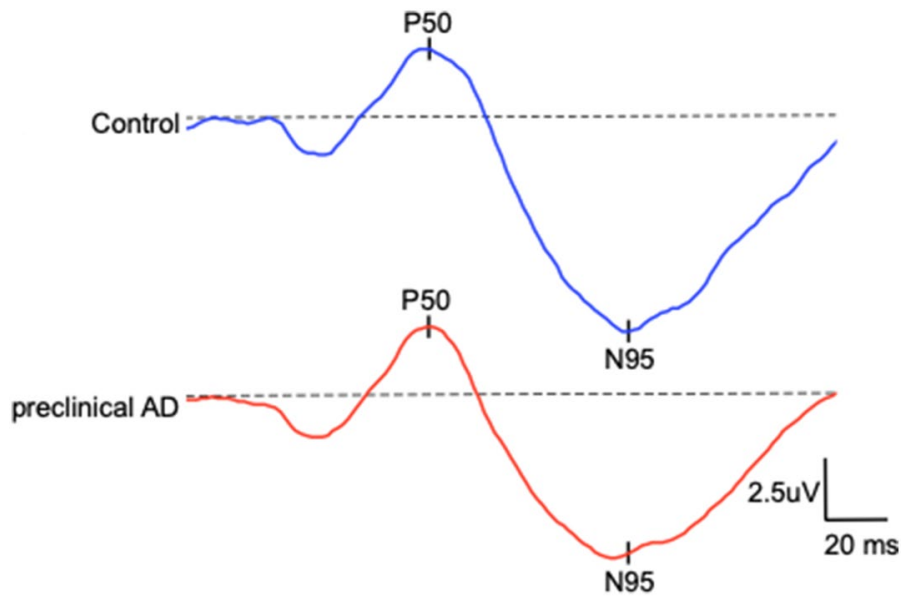

Figure 1. Depicts the recorded pattern electroretinogram responses in representative preclinical Alzheimer's disease $(\mathrm{AD})$ (mean N95 \pm SD: $5.7 \pm 2.3 \mu \mathrm{V}$ ) and control participants (mean N95 \pm SD: $6.1 \pm 1.7 \mu \mathrm{V}$ ).

PERG. Figure 1 depicts the recorded PERG responses in representative $\mathrm{CH}-\mathrm{PATs}$ and $\mathrm{CH}-\mathrm{NATs}$. As shown in Fig. 2, mean statistical analysis showed no statistically significant differences in N95, P50, or N95/P50 ratio between the two groups ( $p>0.05$ for all).

ffERG. When using a $3 \mathrm{~cd}^{*} \mathrm{~s} / \mathrm{m}^{2}$ stimulus intensity, there was a significant delay in the a- $(p=0.006)$ and b-wave components $(p=0.01)$ of the photopic ffERG response in CH-PATs relative to CH-NATs (Table 2). There were no statistically significant differences in a- and b-wave amplitudes or implicit times for the remaining scotopic or photopic ffERG protocols between the two groups ( $p>0.05$ for all).

PhNR. Figure 3 depicts the recorded PhNR responses in representative CH-PATs and CH-NATs. As shown in Fig. 4, PhNR amplitude was significantly decreased in $\mathrm{CH}$-PATs relative to $\mathrm{CH}$-NATs (50.1\% reduction; $p=0.003)$. There was no statistically significant delay in PhNR implicit time between the two groups $(p>0.05)$.

Predicting $\mathrm{CH}-\mathrm{PAT}$ versus $\mathrm{CH}-\mathrm{NAT}$ group classification. Logistic regression was used to predict $\mathrm{CH}-\mathrm{PAT}$ group classification (dependent variable) from ERG indices (independent variables). After running the analysis for all ERG parameters (PERG, ffERG, PhNR, amplitude and implicit time), the strongest model included only PhNR amplitude and N95 implicit time as predictors $(p<0.0001)$. Upon determining the best model (PhNR amplitude and N95 implicit time), a cutoff for predicted event probability was chosen such that sensitivity was at least $85 \%$ and specificity was maximized. The selected cutoff of 0.5 yielded $87 \%$ sensitivity and $82 \%$ specificity $(\mathrm{AUC}=0.84)(95 \%$ CI $0.72-0.95)$ (Fig. 5).

\section{Discussion}

This is the first study to conduct an in vivo electrophysiologic evaluation of the retina in pre-symptomatic AD. We objectively assessed both inner and outer retinal function in human participants by PERG, ffERG, and PhNR analysis. Intriguingly, preclinical AD eyes with normal retinal morphology by OCT exhibited significant retinal dysfunction by ERG relative to controls. We further evaluated the potential diagnostic accuracy of ERG for preclinical AD. Notably, retinal electrophysiologic indices correctly predicted the biochemical class of CH-NAT versus $\mathrm{CH}-\mathrm{PAT}$ in cognitively healthy, older individuals with high sensitivity and specificity. These electrophysiologic findings provide novel insights into early retinal functional changes that precede cognitive decline in AD. Retinal ganglion cell dysfunction may be clinically valuable as a quantitative, objective metric for non-invasive in vivo diagnosis of early disease, which is necessary for ultimately pursuing early intervention.

ERG detected significant RGC dysfunction in pre-symptomatic AD. Previous studies have demonstrated RGC functional deficits in symptomatic AD. Katz et al. first provided electrophysiologic evidence of RGC dysfunction in $\mathrm{AD}$, as reflected by a diminished PERG response in patients relative to healthy controls ${ }^{32}$. Additional studies conducted by Trick et al. and Parisi et al. similarly showed an abnormal PERG response in $\mathrm{AD}^{33,39}$. Recent reports have also detected RGC functional loss in treatment naïve as well as in early stages of AD by PERG ${ }^{35,36}$. Notably, however, studies conducted by Strenn et al. failed to detect significant differences in PERG responses between $\mathrm{AD}$ patients and controls ${ }^{40}$. Discrepancies in ERG findings may be attributed to the challenges of unequivocally diagnosing AD clinically, and also the reduced cooperation typically seen with elderly and cognitively compromised patients. The current study analyzed the PhNR as a measure of inner retinal function. Notably, the PhNR was markedly diminished as compared to the PERG in preclinical AD. The PhNR has shown to be an effective measure of RGC function in optic neuropathies including optic atrophy, optic neuritis, and glaucoma ${ }^{41-43}$. More recently, our laboratory demonstrated the clinical utility of the PhNR in Leber's hereditary optic neuropathy (LHON), whereby the PhNR was not only decreased in affected LHON patients, but also asymptomatic LHON carriers $^{44}$. Compared to PERG, the PhNR is less prone to interfering factors including uncorrected refractive 

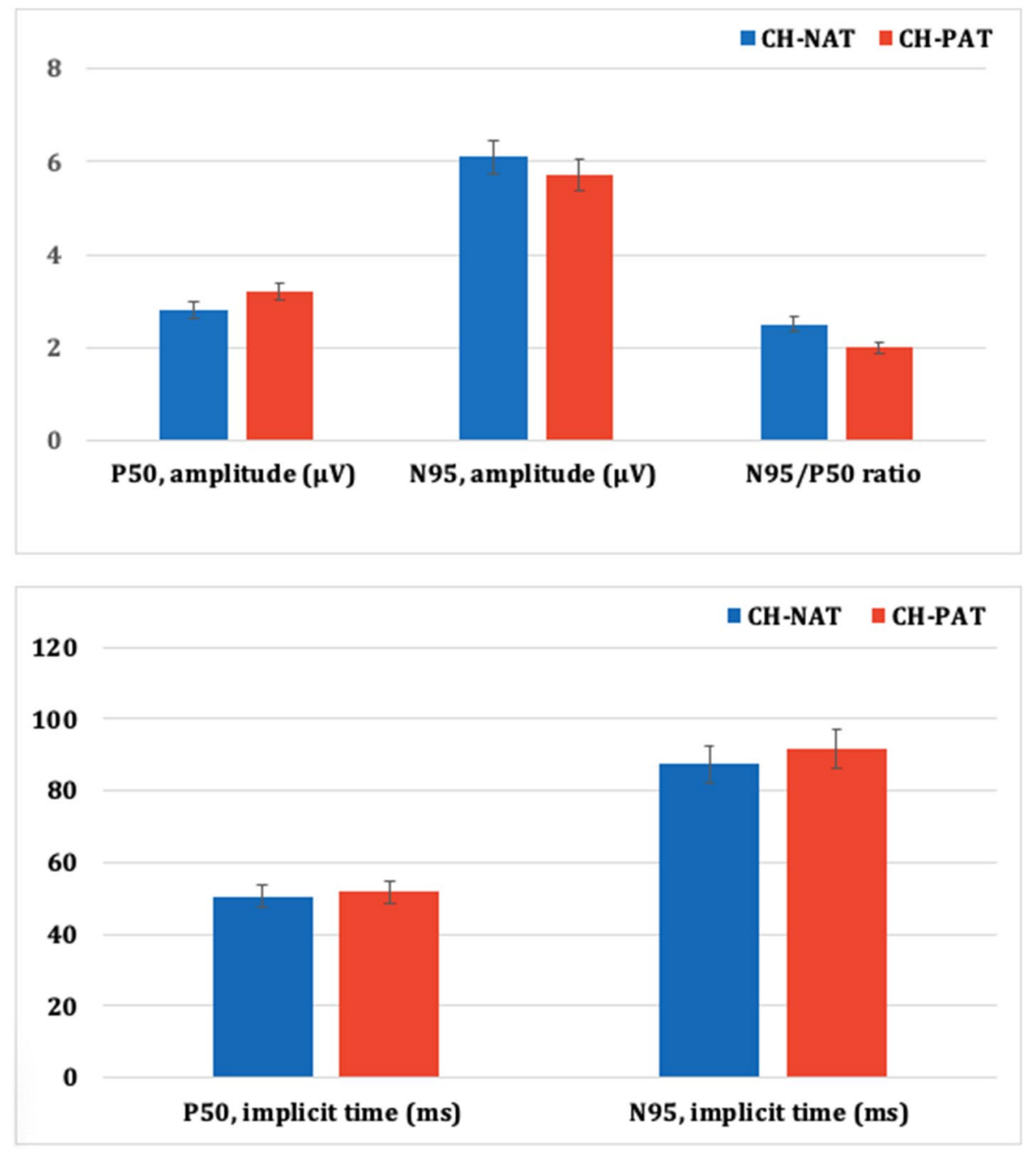

Figure 2. Comparison of the recorded amplitudes (top) and implicit times (bottom) pattern electroretinogram responses between $\mathrm{CH}-\mathrm{NAT}$ and $\mathrm{CH}-\mathrm{PAT}$ participants. $\mathrm{CH}-\mathrm{NAT}$, cognitively healthy with normal amyloid/tau ratio; $\mathrm{CH}-\mathrm{PAT}$, cognitively healthy with pathologic amyloid/tau ratio. Error bars denote standard error.

error or unsteady fixation, as frequently seen with older adults ${ }^{43,45}$. The absence of these constraints in the PhNR allows for a direct measure of RGC physiology. Our ERG findings further support the PhNR as a more robust, objective metric of RGC function and suggest its potential utility as an accessible clinical marker in older adults with pre-symptomatic $\mathrm{AD}$.

Outer retinal function was relatively spared in pre-symptomatic AD. The photopic ffERG was delayed in preclinical $\mathrm{AD}$ when using a $3 \mathrm{~cd}^{*} \mathrm{~s} / \mathrm{m}^{2}$ stimulus intensity. Despite statistical significance, however, we only noted a $\sim 1.5 \mathrm{~ms}$ increase in implicit time which does not suggest clinically significant outer retinal dysfunction of the cone system when compared with reference values from our normative data base and other published data sets ${ }^{46}$. In line with this viewpoint, we also did not observe significant differences in the ffERG photopic or scotopic responses at alternative signal intensities, which overall suggest preserved photoreceptor function in preclinical AD eyes. Our findings are consistent with prior studies in advanced AD disease stages, which have similarly reported preserved outer retinal function in $\mathrm{AD}$, as measured by ffERG ${ }^{32,40}$. This pattern of retinal dysfunction, whereby the inner retina is selectively affected relative to the outer retina, clinically parallels histologic findings reported in $\mathrm{AD}$. In a previous study conducted by our group, postmortem tissues histopathologically confirmed for $\mathrm{A} \beta$ deposition and derived from patients neuropathologically confirmed for severe AD exhibited marked retinal atrophy most pronounced at the inner layers involving the RGCs. These morphological changes corresponded with the distribution of retinal $A \beta$ deposits in these tissues, as previously demonstrated by our laboratory as well as others ${ }^{8,13,47-49}$. These histopathologic results corroborate our retinal electrophysiologic findings and support the premise of primary and early involvement of RGCs in AD.

We further evaluated the discriminatory potential of ERG functional measures for group classification of $\mathrm{CH}-\mathrm{PAT}$ versus $\mathrm{CH}-\mathrm{NAT}$. Higher significance for a given parameter does not automatically imply stronger predictivity, so we examined all ERG indices, as opposed to a priori selection of significant parameters from initial group comparison analysis ${ }^{50}$. Among all ERG parameters, PhNR amplitude together with N95 implicit time constituted a robust model for distinguishing the two cognitively healthy cohorts with high sensitivity and 


\begin{tabular}{|c|c|c|c|}
\hline & CH-NAT & CH-PAT & $\mathbf{P}$ \\
\hline DA $0.01 \mathrm{~cd}^{*} \mathrm{~s} / \mathrm{m}^{2}$ b-wave, amplitude, $\mu \mathrm{V}$ & $253.9 \pm 105.7$ & $222.4 \pm 87.7$ & 0.21 \\
\hline DA $0.01 \mathrm{~cd}^{*} \mathrm{~s} / \mathrm{m}^{2}$ b-wave, implicit time, $\mathrm{ms}$ & $97.2 \pm 15.2$ & $95.7 \pm 13.2$ & 0.73 \\
\hline DA $3 \mathrm{~cd}^{*} \mathrm{~s} / \mathrm{m}^{2}$ a-wave, amplitude, $\mu \mathrm{V}$ & $179.5 \pm 56.0$ & $190.5 \pm 57.4$ & 0.6 \\
\hline DA $3 \mathrm{~cd}^{*} \mathrm{~s} / \mathrm{m}^{2}$ a-wave, implicit time, $\mathrm{ms}$ & $19.8 \pm 2.9$ & $20.3 \pm 3.2$ & 0.65 \\
\hline DA $3 \mathrm{~cd}^{*} \mathrm{~s} / \mathrm{m}^{2}$ b-wave, amplitude, $\mu \mathrm{V}$ & $327.0 \pm 82.3$ & $334.3 \pm 120.1$ & 0.96 \\
\hline DA $3 \mathrm{~cd}^{*} \mathrm{~s} / \mathrm{m}^{2} \mathrm{~b}$-wave, implicit time, $\mathrm{ms}$ & $57.2 \pm 6.1$ & $57.7 \pm 10.0$ & 0.71 \\
\hline DA $10 \mathrm{~cd}^{*} \mathrm{~s} / \mathrm{m}^{2}$ a-wave, amplitude, $\mu \mathrm{V}$ & $229.6 \pm 70.6$ & $229.0 \pm 79.3$ & 0.91 \\
\hline DA $10 \mathrm{~cd}^{*} \mathrm{~s} / \mathrm{m}^{2}$ a-wave, implicit time, $\mathrm{ms}$ & $14.8 \pm 1.1$ & $15.2 \pm 1.8$ & 0.46 \\
\hline DA $10 \mathrm{~cd}^{*} \mathrm{~s} / \mathrm{m}^{2} \mathrm{~b}$-wave, amplitude, $\mu \mathrm{V}$ & $133.6 \pm 62.7$ & $132.5 \pm 55.8$ & 0.67 \\
\hline DA $10 \mathrm{~cd}^{\star} \mathrm{s} / \mathrm{m}^{2}$ b-wave, implicit time, $\mathrm{ms}$ & $58.5 \pm 5.4$ & $59.1 \pm 5.7$ & 0.59 \\
\hline LA $3 \mathrm{~cd}^{*} \mathrm{~s} / \mathrm{m}^{2}$ a-wave, amplitude, $\mu \mathrm{V}$ & $29.0 \pm 21.2$ & $33.0 \pm 17.1$ & 0.47 \\
\hline LA $3 \mathrm{~cd}^{*} \mathrm{~s} / \mathrm{m}^{2}$ a-wave, implicit time, $\mathrm{ms}$ & $14.1 \pm 1.8$ & $15.5 \pm 1.7$ & 0.006 \\
\hline LA $3 \mathrm{~cd}^{*} \mathrm{~s} / \mathrm{m}^{2} \mathrm{~b}$-wave, amplitude, $\mu \mathrm{V}$ & $91.3 \pm 30.5$ & $106.1 \pm 37.2$ & 0.17 \\
\hline LA $3 \mathrm{~cd}^{\star} \mathrm{s} / \mathrm{m}^{2}$ b-wave, implicit time, $\mathrm{ms}$ & $31.9 \pm 1.2$ & $33.5 \pm 2.7$ & 0.01 \\
\hline LA $30 \mathrm{~Hz}$ flicker, amplitude, $\mu \mathrm{V}$ & $74.8 \pm 39.4$ & $90.1 \pm 36.0$ & 0.19 \\
\hline LA $30 \mathrm{~Hz}$ flicker, implicit time, ms & $28.7 \pm 2.2$ & $30.2 \pm 2.5$ & 0.06 \\
\hline
\end{tabular}

Table 2. Waveform analysis results of the full-field electroretinogram in $\mathrm{CH}-\mathrm{NAT}$ and $\mathrm{CH}-\mathrm{PAT}$. Illustrates amplitude and implicit times of the dark-(DA) and light-adapted (LA) retina in response to ISCEV-defined stimulus flash intensities $\left(\mathrm{cd}^{\star} \mathrm{s} / \mathrm{m}^{2}\right)$. Abbreviations: CH-NAT, cognitively healthy with normal amyloid/tau ratio; $\mathrm{CH}-\mathrm{PAT}$, cognitively healthy with pathologic amyloid/tau ratio. Data provided as mean \pm standard deviation.

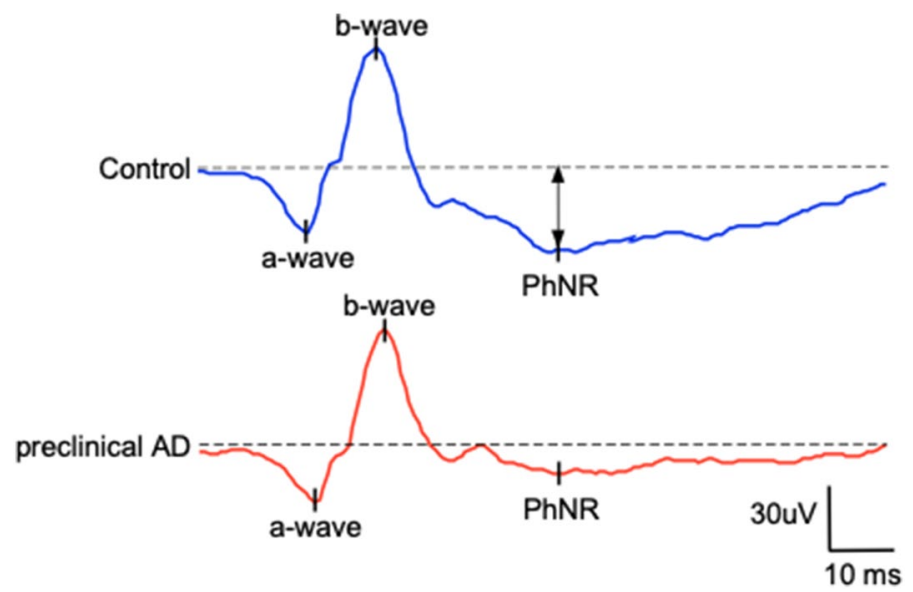

Figure 3. Depicts the recorded photopic negative responses (PhNR) in representative preclinical Alzheimer's disease $(\mathrm{AD})$ and control participants. As illustrated by the double-headed arrow, the PhNR was markedly diminished in preclinical AD as compared to controls.

specificity. Previous studies demonstrating N95 prolongation in patients with symptomatic AD further supports the potential predictive value of this parameter ${ }^{34,36}$. These findings suggest that retinal electrophysiologic data have sufficient sensitivity and specificity to differentiate patients with CSF biomarkers of AD pathology.

We recently conducted a prospective study evaluating retinal structural morphology by OCT in CH-PAT and $\mathrm{CH}-\mathrm{NAT}$ participants ${ }^{30}$. Preclinical AD individuals exhibited significant retinal thinning of the RNFL, which represents the axonal fibers connecting the ganglion cells to the brain. Notably, RNFL thickness classified CHNAT versus $\mathrm{CH}-\mathrm{PAT}$ with $87 \%$ sensitivity and $56.3 \%$ specificity $^{31}$. The current study evaluated retinal function in premanifest AD participants with in vivo normal retinal thickness. ERG detected significant RGC dysfunction, despite normal structural morphology by OCT, and yielded a superior diagnostic performance (87\% sensitivity and $82 \%$ specificity). Our findings of ERG abnormalities in the absence of in vivo retinal thinning suggests the notion that functional changes precede structural changes in $\mathrm{AD}$, as indicated by previous reports ${ }^{36}$. Alternatively, retinal functional deficits assessed by ERG are more likely to be measured before detectable structural changes by OCT.

Limitations of this study include those associated with an exploratory approach. The statistical models await validation, and the findings should be replicated and applied to different populations to assess both neurological and ophthalmological disease, physiological, and cultural/ethnic specificity. Nevertheless, the present article 


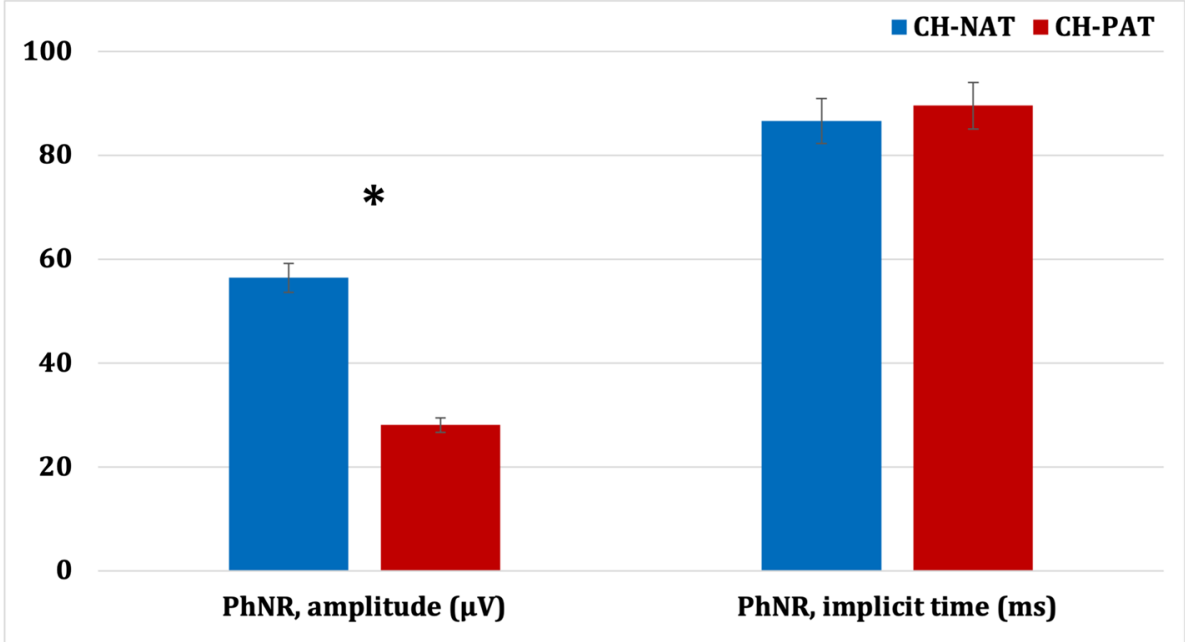

Figure 4. Photopic negative response (PhNR) comparison between $\mathrm{CH}-\mathrm{NAT}$ and $\mathrm{CH}-\mathrm{PAT}$ participants. $\mathrm{CH}-\mathrm{NAT}$, cognitively healthy with normal amyloid/tau ratio; $\mathrm{CH}-\mathrm{PAT}$, cognitively healthy with pathologic amyloid/tau ratio. Error bars denote standard error, ${ }^{*} p<0.05$.

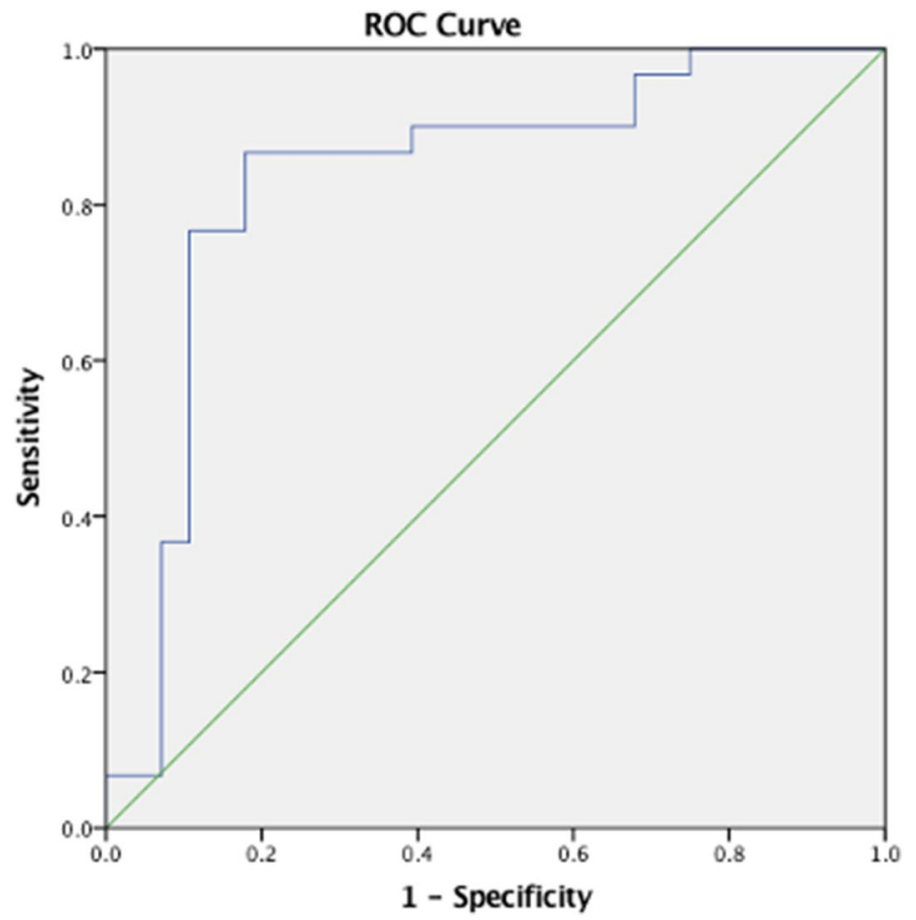

Figure 5. Representation of the ROC curve of the regression analysis to demonstrate which parameters of ERG predict $\mathrm{CH}-\mathrm{NAT}$ versus $\mathrm{CH}-\mathrm{PAT}$ group classification. The area under the curve (AUC) of PhNR amplitude and N95 implicit time was 0.84 .

represents the first electrophysiologic assessment of the retina in preclinical $\mathrm{AD}$. In addition, preclinical AD and control participants were specifically classified according to $A \beta_{42} /$ Tau. This classification scheme further strengthens the significance of our findings given the superior sensitivity and specificity of $A \beta_{42} /$ Tau relative to the CSF concentration of these biomarkers alone ${ }^{30}$. Glaucoma is another common ocular disease that has been implicated as sharing commonalities with $\mathrm{AD}^{47}$. However, the relationship between these two disease entities remains controversial and additional studies are warranted to investigate their similarities. Importantly, all participants enrolled in our study underwent complete ophthalmic examination to exclude potentially confounding ocular pathologies. The current study did not analyze axial length. Retinal stretching and cell damage associated with axial elongation could lead to retinal thinning and diminished ERG amplitude. However, these 
effects of axial myopia are most notable in high myopes ( $>6$ diopters $)^{51}$. In the present article, participants with refractive error $>5$ diopters were excluded and there were no significant differences in OCT retinal thickness between cohorts. While delayed implicit time has also been observed with increasing axial length, these effects are reportedly less remarkable ${ }^{51}$. Given the cross-sectional nature of our investigation, long-term longitudinal follow-up studies are necessary for the proper characterization of disease natural history. In turn, we plan to use these results as baseline values for future longitudinal studies. Further research is also necessary to precisely elucidate the mechanism of retinal pathology observed in these cohorts. Additional studies investigating the relationship between electrophysiology and alternative measures of retinal function including visual field performance testing may be particularly insightful. Nevertheless, this study suggests the utility of the PhNR as a reliable, objective and sensitive metric that directly reflects RGC physiology in presymptomatic AD. In addition, retinal electrophysiologic data, namely the PhNR and N95, may allow for improved diagnostics of AD pathology before cognitive decline, as compared to OCT structural data alone. These are important outcome measure against which to evaluate the efficacy of purported treatment strategies for AD.

Received: 5 November 2020; Accepted: 20 February 2021

Published online: 18 March 2021

\section{References}

1. Alzheimer's Association,. Alzheimer's disease facts and figures. Alzheimers Dement. 16, 391-460 (2020).

2. Batsch, N. L. \& Mittelman, M. S. World Alzheimer Report 2012: Overcoming the Stigma of Dementia Vol. 5 (Alzheimer's Disease International, London, 2012).

3. McKhann, G. et al. Clinical diagnosis of Alzheimer's disease: Report of the NINCDS-ADRDA Work Group* under the auspices of Department of Health and Human Services Task Force on Alzheimer's Disease. Neurology 34, 939-939 (1984).

4. Katz, B. \& Rimmer, S. Ophthalmologic manifestations of Alzheimer's disease. Surv. Ophthalmol. 34, 31-43 (1989).

5. Tzekov, R. \& Mullan, M. Vision function abnormalities in Alzheimer disease. Surv. Ophthalmol. 59, 414-433 (2014).

6. Thomson, K. L., Yeo, J. M., Waddell, B., Cameron, J. R. \& Pal, S. A systematic review and meta-analysis of retinal nerve fiber layer change in dementia, using optical coherence tomography. Alzheimer's Dement. Diagn. Assess. Dis. Monit. 1, 136-143 (2015).

7. Dugger, B. N., Tu, M., Murray, M. E. \& Dickson, D. W. Disease specificity and pathologic progression of tau pathology in brainstem nuclei of Alzheimer's disease and progressive supranuclear palsy. Neurosci. Lett. 491, 122-126 (2011).

8. Sadun, A. A., Borchert, M., DeVita, E., Hinton, D. R. \& Bassi, C. J. Assessment of visual impairment in patients With Alzheimer's disease. Am. J. Ophthalmol. 104, 113-120 (1987).

9. Javaid, F. Z., Brenton, J., Guo, L. \& Cordeiro, M. F. Visual and ocular manifestations of Alzheimer's disease and their use as biomarkers for diagnosis and progression. Front. Neurol. 7, 55 (2016).

10. La Morgia, C., Di Vito, L., Carelli, V. \& Carbonelli, M. Patterns of retinal ganglion cell damage in neurodegenerative disorders: Parvocellular vs magnocellular degeneration in optical coherence tomography studies. Front. Neurol. 8, 710 (2017).

11. Uhlmann, R. F., Larson, E. B., Koepsell, T. D., Rees, T. S. \& Duckert, L. G. Visual impairment and cognitive dysfunction in Alzheimer's disease. J Gen Intern Med 6, 126-132 (1991).

12. Hinton, D. R., Sadun, A. A., Blanks, J. C. \& Miller, C. A. Optic-nerve degeneration in Alzheimer's disease. N Engl J Med 315, 485-487 (1986).

13. La Morgia, C. et al. Melanopsin retinal ganglion cell loss in Alzheimer disease. Ann Neurol. 79, 90-109 (2016).

14. Blanks, J. C., Hinton, D. R., Sadun, A. A. \& Miller, C. A. Retinal ganglion cell degeneration in Alzheimer's disease. Brain Res. 501, 364-372 (1989).

15. Blanks, J. C., Torigoe, Y., Hinton, D. R. \& Blanks, R. H. I. Retinal degeneration in the macula of patients with Alzheimer's disease. Ann. N. Y. Acad. Sci. 640, 44-46 (1991).

16. Blanks, J. C., Torigoe, Y., Hinton, D. R. \& Blanks, R. H. I. Retinal pathology in Alzheimer's disease. I. Ganglion cell loss in foveal/ parafoveal retina. Neurobiol. Aging 17, 377-384 (1996).

17. Blanks, J. C. et al. Retinal pathology in Alzheimer's disease. II. Regional neuron loss and glial changes in GCL. Neurobiol. Aging 17, 385-395 (1996).

18. Curcio, C. A. \& Drucker, D. N. Retinal ganglion cells in Alzheimer's disease and aging. Ann Neurol. 33, 248-257 (2004).

19. Garcia-Martin, E. et al. Neuro-ophthalmologic evaluation, quality of life, and functional disability in patients with MS. Neurology 81, 76-83 (2013).

20. Garcia-Martin, E. et al. Ganglion cell layer measurements correlate with disease severity in patients with Alzheimer's disease. Acta Ophthalmol 94, e454-e459 (2016).

21. Liu, D. et al. Thinner changes of the retinal nerve fiber layer in patients with mild cognitive impairment and Alzheimer's disease. BMC Neurol 15, 14 (2015).

22. Chan, V. T. T. et al. Spectral-domain OCT measurements in Alzheimer's disease. Ophthalmology 126, 497-510 (2019).

23. Coppola, G. et al. Optical coherence tomography in Alzheimer's disease: A meta-analysis. PLoS ONE 10, e0134750 (2015).

24. Shen, Y. et al. The attenuation of retinal nerve fiber layer thickness and cognitive deterioration. Front. Cell. Neurosci. 7, 142 (2013).

25. Shen, Y. et al. Retinal nerve fiber layer thickness is associated with episodic memory deficit in mild cognitive impairment patients. Curr Alzheimer Res 11, 259-266 (2014).

26. Shi, Z. et al. Greater attenuation of retinal nerve fiber layer thickness in Alzheimer's disease patients. JAD 40, 277-283 (2014).

27. Kromer, R. et al. Detection of retinal nerve fiber layer defects in Alzheimer's disease using SD-OCT. Front. Psychiatry 5, 22 (2014).

28. Ascaso, F. J. et al. Retinal alterations in mild cognitive impairment and Alzheimer's disease: An optical coherence tomography study. J. Neurol. 261, 1522-1530 (2014).

29. Tapiola, T. et al. Cerebrospinal fluid $\beta$-amyloid 42 and tau proteins as biomarkers of Alzheimer-type pathologic changes in the brain. Arch. Neurol. 66, 382-389 (2009).

30. Harrington, M. G. et al. Executive function changes before memory in preclinical Alzheimer's pathology: A prospective, crosssectional, case control study. PLoS ONE 8, e79378 (2013).

31. Asanad, S. et al. Retinal nerve fiber layer thickness predicts CSF amyloid/tau before cognitive decline. PLoS ONE 15, e0232785 (2020).

32. Katz, B., Rimmer, S., Iragui, V. \& Katzman, R. Abnormal pattern electroretinogram in Alzheimer's disease: Evidence for retinal ganglion cell degeneration?. Ann Neurol. 26, 221-225 (1989).

33. Trick, G. L., Barris, M. C. \& Bickler-Bluth, M. Abnormal pattern electroretinograms in patients with senile dementia of the alzheimer type. Ann Neurol. 26, 226-231 (1989).

34. Parisi, V. et al. Morphological and functional retinal impairment in Alzheimer's disease patients. Clin. Neurophysiol. 112, $1860-1867$ (2001). 
35. Sartucci, F. et al. Dysfunction of the magnocellular stream in Alzheimer's disease evaluated by pattern electroretinograms and visual evoked potentials. Brain Res. Bull. 82, 169-176 (2010).

36. Krasodomska, K., Lubiński, W., Potemkowski, A. \& Honczarenko, K. Pattern electroretinogram (PERG) and pattern visual evoked potential (PVEP) in the early stages of Alzheimer's disease. Doc Ophthalmol 121, 111-121 (2010).

37. Mwanza, J.-C. et al. Macular ganglion cell-inner plexiform layer: Automated detection and thickness reproducibility with spectral domain-optical coherence tomography in glaucoma. Invest. Ophthalmol. Vis. Sci. 52, 8323 (2011).

38. Hu, J. et al. Improved repeatability of retinal thickness measurements using line-scan ophthalmoscope image-based retinal tracking. Ophthalmic Surg. Lasers Imaging Retina 46, 310-314 (2015).

39. Parisi, V. et al. Correlation between optical coherence tomography, pattern electroretinogram, and visual evoked potentials in open-angle glaucoma patients. Ophthalmology 108, 905-912 (2001).

40. Strenn, K. et al. Pattern electroretinogram and luminance electroretinogram in Alzheimer's disease. J. Neural. Transm. Suppl. 33, 73-80 (1991).

41. Viswanathan, S., Frishman, L. J., Robson, J. G. \& Walters, J. W. The photopic negative response of the flash electroretinogram in primary open angle glaucoma. Invest. Ophthalmol. Vis. Sci. 42, 514-522 (2001).

42. Nakamura, H., Miyamoto, K., Yokota, S., Ogino, K. \& Yoshimura, N. Focal macular photopic negative response in patients with optic neuritis. Eye 25, 358-364 (2011).

43. Viswanathan, S., Frishman, L. J., Robson, J. G., Harwerth, R. S. \& Smith, E. L. The photopic negative response of the macaque electroretinogram: reduction by experimental glaucoma. Invest. Ophthalmol. Vis. Sci. 40, 1124-1136 (1999).

44. Karanjia, R. et al. The photopic negative response: An objective measure of retinal ganglion cell function in patients with Leber's hereditary optic neuropathy. Invest. Ophthalmol. Vis. Sci. 58, BIO300 (2017).

45. Moss, H. E., Park, J. C. \& McAnany, J. J. The photopic negative response in idiopathic intracranial hypertension. Invest. Ophthalmol. Vis. Sci. 56, 3709 (2015).

46. Parvaresh, M.-M., Ghiasian, L., Ghasemi Falavarjani, K., Soltan Sanjari, M. \& Sadighi, N. Normal values of standard full field electroretinography in an Iranian population. J. Ophthalmic Vis. Res. 4, 97-101 (2009).

47. Hart, N. J., Koronyo, Y., Black, K. L. \& Koronyo-Hamaoui, M. Ocular indicators of Alzheimer's: exploring disease in the retina. Acta Neuropathol 132, 767-787 (2016).

48. Koronyo-Hamaoui, M. et al. Identification of amyloid plaques in retinas from Alzheimer's patients and noninvasive in vivo optical imaging of retinal plaques in a mouse model. Neuroimage 54, S204-S217 (2011).

49. Asanad, S. et al. The retina in Alzheimer's disease: Histomorphometric analysis of an ophthalmologic biomarker. Invest. Ophthalmol. Vis. Sci. 60, 1491 (2019).

50. Lo, A., Chernoff, H., Zheng, T. \& Lo, S.-H. Why significant variables aren't automatically good predictors. Proc. Natl. Acad. Sci. U.S.A 112, 13892-13897 (2015).

51. Sachidanandam, R., Ravi, P. \& Sen, P. Effect of axial length on full-field and multifocal electroretinograms: Effect of axial length on full-field and multifocal ERGs Sachidanandam, Ravi and Sen. Clin. Exp. Optom. 100, 668-675 (2017).

\section{Acknowledgement}

We thank our funding sources including Research to Prevent Blindness, Inc. (RPB), the National Institute of Aging (NIA), and the International Foundation for Optic Nerve Diseases (IFOND).

\section{Author contributions}

S.A: Study design, data collection, data analysis, data interpretation; manuscript preparation; C.M.F: Data collection, manuscript review; M.F: Data collection; M.G.H: Study design, data collection, manuscript review; A.A.S: Study design, data interpretation, manuscript review; R.K: Study design, data interpretation, manuscript review.

\section{Funding}

This study was funded by the Brain Aging Study from the L.K. Whittier Foundation (to MGH); NIH National Institute on Aging grant \# P50-AG05142 (USC ADRC Neuropathology Core Grant); Research to Prevent Blindness, Inc. (unrestricted grant), and the International Foundation for Optic Nerve Diseases (IFOND).

\section{Competing interests}

The authors declare no competing interests.

\section{Additional information}

Correspondence and requests for materials should be addressed to S.A.

Reprints and permissions information is available at www.nature.com/reprints.

Publisher's note Springer Nature remains neutral with regard to jurisdictional claims in published maps and institutional affiliations.

(c) (i) Open Access This article is licensed under a Creative Commons Attribution 4.0 International cc. License, which permits use, sharing, adaptation, distribution and reproduction in any medium or format, as long as you give appropriate credit to the original author(s) and the source, provide a link to the Creative Commons licence, and indicate if changes were made. The images or other third party material in this article are included in the article's Creative Commons licence, unless indicated otherwise in a credit line to the material. If material is not included in the article's Creative Commons licence and your intended use is not permitted by statutory regulation or exceeds the permitted use, you will need to obtain permission directly from the copyright holder. To view a copy of this licence, visit http://creativecommons.org/licenses/by/4.0/.

(c) The Author(s) 2021 